\title{
Analysis on clinical features of death patients with COVID-19: a retrospective, single-center study from Wuhan, China
}

\section{Ke Yao}

Tongji Hospital of Tongji Medical College of Huazhong University of Science and Technology

\section{Yin Zhao}

Tongji Hospital of Tongji Medical College of Huazhong University of Science and Technology Department of Radiology

\section{Xiangtian Xiao}

Wuhan University of Science and Technology

\section{Gunyun Wu}

Tongji Hospital of Tongji Medical College of Huazhong University of Science and Technology

\section{Ruxin Xie}

Tongji Hospital of Tongji Medical College of Huazhong University of Science and Technology Department of Radiology

\section{Yali Wang}

Tongji Hospital of Tongji Medical College of Huazhong University of Science and Technology Department of Radiology

Jun $\mathrm{Hu}$

Tongji Hospital of Tongji Medical College of Huazhong University of Science and Technology Hexie Cai

Tongji Hospital of Tongji Medical College of Huazhong University of Science and Technology Rong Liu ( $\sim$ rongr007@outlook.com )

\section{Research article}

Keywords: Severe acute respiratory syndrome coronavirus 2 (SARS-CoV-2), Coronavirus disease 2019 (COVID-19), High-sensitive cardiac troponin I

Posted Date: April 25th, 2020

DOI: https://doi.org/10.21203/rs.3.rs-21065/v1

License: (c) (i) This work is licensed under a Creative Commons Attribution 4.0 International License. Read Full License 


\section{Abstract}

Background An ongoing global pandemic of pneumonia caused by severe acute respiratory syndrome coronavirus 2 (SARS-CoV-2) has caused thousands of deaths in China since December, 2019. We aimed to describe the clinical course of patients died of SARS-CoV-2 infection.

Methods In this retrospective study, we reviewed 194 patients with SARS-CoV-2 infection, who died consecutively between Feb 3 to 24, 2020 in Tongji Hospital (Wuhan, China). Basic demographic and clinical information, laboratory findings, complications and treatments were extracted from electronic medical records of Hospital Information System. Unpaired t test was employed to evaluate the statistical differences of the serum level of high-sensitive cardiac troponin I (hs-cTnl) among different age or sex groups. The relationship between hs-cTnl and inflammatory cytokines were estimated using Spearman correlation analysis.

Results The death patients aged $69.62 \pm 10.98$, in which $68.6 \%$ were male. $74.7 \%$ patients had underlying chronic illnesses. The most common symptoms were fever (83\%), cough (69.3\%), and dyspnea (65.6\%). Decreased lymphocyte count (91.4\%), elevated level of hs-cTnl (82.9\%) and inflammatory parameters in serum were commonly seen. The hs-cTnl level was significantly higher in the group aged 60-79 and male patients. A week positive correlation was observed between hs-cTnl values and D-dimer values $(r=0.343$, $\mathrm{p} \otimes 0.05)$. Acute respiratory distress syndrome was the main complication. Assisted respiration, antimicrobial drugs, glucocorticoids and immune globulin were the major treatments.

Conclusion Most non-survivors with SARS-CoV-2 infection were old with chronic illnesses, complicated by multiple organ dysfunction. Prevention is better than cure in high-risk population.

\section{Background}

Severe acute respiratory syndrome coronavirus 2 (SARS-CoV-2) pneumonia caused by a novel enveloped RNA beta-coronavirus were identified in the city of Wuhan since early Dec, 2019.(1) The World Health Organization recently named it as coronavirus disease 2019 (COVID-19) and special control measures have been taken to prevent transmission.(2) Till Mar 9, 2020, there were a total of 3123 death cases in 80904 confirmed patients in China (3.86\% death rate) and 484 death cases in 24727 confirmed patients in the rest world ( $1.96 \%$ death rate). Given the rapid spread and a large amount of infected patients, COVID-19 has raised an international concern.

COVID-19 has characteristic laboratory findings and chest computed tomography (CT) abnormalities. (3) However, the data on the clinical characteristics of patients died of COVID-19 are scarce. In this study, we summarized the clinical characteristics of 194 patients died from COVID-19, who were treated in Tongji hospital, Wuhan, China. We hope that an updated analysis of these data could help to raise warning and reduce mortality under SARS-CoV-2 epidemic condition.

\section{Methods}




\section{Patients}

194 patients died of COVID-19 consecutively from Feb 03 to 24, 2020 were collected from the Sino-French Branch of Tongji Hospital, which is one of the major tertiary healthcare system and teaching hospitals in in Wuhan, Hubei Province, and responsible for the treatments of severe SARS-CoV-2 infection designated by the Chinese government. All patients with COVID-19 enrolled in this study were diagnosed according to the WHO interim guideline. (4)

\section{Data collection}

Clinical characteristics and laboratory test results of 194 non-survival patients with COVID-19 were extracted from the electronic medical records of Hospital Information System. All available medical history, symptoms and signs, underlying diseases, laboratory results, treatments and complications were recorded. The underlying diseases mainly included hypertension, diabetes, cardiovascular diseases, chronic pulmonary diseases, hepatitis, cerebrovascular diseases, malignancies, chronic kidney diseases, major surgeries in recent three months. Laboratory test results mainly included peripheral blood cell counts, blood biochemical tests (biomarkers of liver and kidney function, and acute cardiac injury), coagulation function and inflammatory cytokines. The complications (acute respiratory distress syndrome (ARDS), acute cardiac injury, arrhythmia, acute kidney injury, liver dysfunction, septic shock, disseminated intravascular coagulation (DIC), brain injury) were also recorded. ARDS were defined under the guidance of WHO for novel COVID-19. (4) Acute kidney injury was identified based on the levels of serum creatinine and estimated glomerular filtration rate. The upper limit of serum concentration of hscTnl was $15.6 \mathrm{pg} / \mathrm{mL}$, measured in clinical laboratory department of Tongji Hospital. Values of hs-cTnl $\geq$ $10000.0 \mathrm{pg} / \mathrm{mL}$ were all recorded as $10000.0 \mathrm{pg} / \mathrm{mL}$. Cardiac injury was identified when hs-cTnl values were elevated more than twice the upper limit. Besides, all hs-cTnl values of the patients tested 14 days before death was also extracted and displayed. Treatments including oxygen inhalation, mechanical ventilation, antiviral, antibacterial and antifungal medication, systemic glucocorticoids, intravenous immune globulin, extracorporeal membrane oxygenation (ECMO) support, and continuous renal replacement therapy (CRRT) were recorded for all patients.

\section{Statistical analysis}

GraphPad Prim (Version 8.0, GraphPad Software Inc., CA, USA) software was used for statistical analysis of the data. Continuous variables were expressed as means \pm standard deviations (SD). Categorical variables were summarized as the counts and percentages. The relationship between hs-cTnl values and inflammatory biomarkers/cytokines (including D-dimer, hs-CRP, ESR, IL-1 $\beta$, IL-2R, IL-6, IL-8, IL-10 and TNFa) were estimated using Spearman correlation analysis. After last recorded hs-cTnl results of each patient were used normalized to fold of hs-cTnl upper limit values $(15.6 \mathrm{pg} / \mathrm{mL})$ as Y-axis, unpaired $t$ test was applied to evaluate the differences of the levels of hs-cTnl values (folds of upper limit) among different age and sex groups. A $p$ value less than 0.05 was considered statistically significant (two-tailed). 


\section{Results}

\section{Demographic and clinical characteristics}

A total of 194 death patients with COVID-19 were included in the present study. The demographics and baseline characteristics were shown in Table 1. The average age of patients was $69.62 \pm 10.98$ years, and $133(68.6 \%)$ were male. Of these patients, $145(74.7 \%)$ had at least one of following chronic illnesses: hypertension (92 [63.4\%]), diabetes (40 [27.5\%]), cardiovascular diseases $\quad(39[26.9 \%])$, chronic pulmonary diseases $\quad(18[12.4 \%])$, chronic hepatitis $(10[6.9 \%])$, cerebrovascular diseases (9[6.2\%]), malignances (9 [6.2\%]), chronic kidney diseases (9 [6.2\%]). $66(45.5 \%)$ patients had more than two kinds of these chronic medical illnesses.

The most common symptoms on admission were fever (83\%), cough (69.3\%), and dyspnea (65.6\%). Malaise, myalgia and chills or fatigue were also seen in patients with incidences of $30.2 \%, 14.9 \%$ and $14.1 \%$ respectively. In addition, 39 of $194(20.1 \%)$ patients complained of diarrhea, $5(2.6 \%)$ showed abdominal pain, and $39(20.1 \%)$ experienced vomiting, which meant the digestive system was involved on the course of COVID-19. Besides, consciousness disorder occurred in 14 (7.2\%) and palpitation occurred in 5 (2.6\%) patients, which hinted that the disease might have affected the central nervous system and cardiac system.

\section{Laboratory findings}

Among the 194 cases, 185 had laboratory test results and the last test results for each parameter before death were recorded and showed in Table 2 . The increased neutrophil $\left(12.84 \pm 8.56 \times 10^{9} / \mathrm{L}\right)$ and decreased lymphocyte count $\left(0.58 \pm 0.42 \times 10^{9} / \mathrm{L}\right)$ in blood were commonly observed. Nearly half of the patients had elevated ALT and AST test values. Abnormal hs-cTnl and NT-proBNP levels occurred in $82.9 \%$ and $91.1 \%$ patients. Besides, the other abnormal results included the increased levels of hs-CRP (100\%), IL-6 (99.1\%) and D-dimer (98.9\%) etc.

The serum level of hs-cTnl is an important indicator of myocardial damage and usually used for diagnosing myocardial infarction. (5) We tracked all the results of hs-cTnl tested during 14 days before death. As shown in Figure 1A, the hs-cTnl level began to rise beyond the normal range from Day 13, and peaked at Day 1 with an average value of $1311 \pm 2762 \mathrm{pg} / \mathrm{mL}$.

For different age groups, there were significant differences between the group younger than $49(10.7 \pm 8.4)$ and the other different age groups. The group aged 60-69 years and 70-79 years both had significant higher levels of hs-cTnl than the group aged $50-59$ years $(37.9 \pm 79.8)$. Besides, there was no significant difference in hs-cTnl levels between the group aged 60-69 years (99.9 206.2 ) and the group aged 70-79 years (78.8 \pm 169.1$)$, meanwhile both groups has notably higher hs-cTnl levels than the group over 80 (37.2 \pm 98.0$)$.(Figure 1B) For different sex groups, male patients tended to suffer from apparently higher hs-cTnl levels $(83.5 \pm 183.4)$ than female patients $(49.5 \pm 112.3)$. (Figure 1C) 


\section{Correlations between hs-cTnl and inflammatory parameters}

There were 141 (82.9\%) patients with abnormal hs-cTnl levels. As shown in Table 3, the inflammatory cytokines were out of the normal range in $20.9 \%-99.1 \%$ patients. Over $95 \%$ patients had abnormal ESR, hs-CRP and D-dimer levels. There was a week positive correlation between hs-cTnl and D-dimer levels with a correlation coefficient of $0.343(p<0.0001)$. The correlations were of no significances between hscTnl and ESR, hs-CRP, IL-1 $\beta$, IL-6, IL-2R, IL-8, IL-10 and TNF-a levels (all $p$ values>0.05; Supplementary Table 1).

\section{Complications and treatments}

Specific complications were documented in 110 (110/194 [55.4\%]) patients according to the electronic medical records. (Table 3)ARDS occurred in $62.7 \%$ patients, which was the major cause of death. Besides, $45.4 \%$ patients underwent cardiac injury with four patients diagnosed as fulminant myocarditis. Other severe complications included acute kidney injury (14.5\%), septic shock $(9.1 \%)$ and DIC $(11.8 \%)$. Moreover, 56 of 110 death patients developed two or more kinds of these complications.

All patients received supportive treatments as soon as admitted into the hospital. $67.5 \%$ patients were treated with oxygen support and $80.9 \%$ underwent mechanical ventilation. $70.1 \%$ patients received antiviral drugs and $87.6 \%$ were empirically given antibacterial medication. Besides, systemic glucocorticoids (e.g., methylprednisolone $40-80 \mathrm{mg}$ ) and intravenous immune globulin were daily used in $90 \%$ and $40.7 \%$ patients, respectively. Two patients underwent ECMO support and four patients underwent CRRT therapy.

\section{Discussion}

The death patients aged $69.62 \pm 10.98$, in which $68.6 \%$ were male. Chronic medical illnesses were complained of by over $2 / 3$ of the cases. ARDS was the main complication of critically severe SARS-CoV- 2 infection. Besides, decreased lymphocyte count, elevated level of hs-cTnl and inflammatory parameters in serum were commonly seen.

In China Medical Treatment Expert Group for Covid-19 report, Guan et al included 1099 cases and divided into non-severe group and severe group. (6) The average age is 45 in non-severe group, and 52 in severe group. In Yang's study (another medical center for COVID-19 in Wuhan), they included 52 cases and divided into survivor group and non-survivor group. (7) They found that the average age is 51.9 in survivor group and 64.6 in non-survivor group. In our study, we reviewed 194 death cases and we found that the average age is 69.6 , older than the previous reported non-survivor group. The amount of male cases was twice as much as female in our study, in accordance with previously reported sex difference. (6)(7) Thus, we inferred that older age and male were risk factors for severe or non-survival condition in COVID-19. 
In Guan's study, they reported that $21 \%$ non-severe patients, and $38.7 \%$ severe patients with chronic coexisting illness. (6) In Yang's study, they reported 20\% survivor, and 53\% non-survivor patients with chronic illness. (7) We found that $74.7 \%$ of the deaths had chronic medical illness. Most importantly, there were $45.5 \%$ of them with more than 2 kinds of the chronic illness. $62.7 \%$ of the patients in our cases developed with an ARDS, while the rest died from other organ or system failure due to the chronic medical illness or secondary infection. Poor physical condition was important risk factors for non-survival.

Chen $\mathrm{C}$ etc. found that COVID-19 can significantly affect the heart function and lead to myocardial injury. (8) The hs-cTnl assays has greatly enhanced the accuracy and efficiency of cardiac injury since 2010. (9, 10) Chen $C$ etc. concluded that the increased level of cTnl is independent determinants clinical disease status in patients with COVID-19 by using univariate logistic regression analysis between mild cases and cases in critical care.(8) We noticed that nearly half of the patients in our study got cardiac injury with hscTnl even up to hundreds of times higher than the upper limit. Besides, the inflammatory response was reported to take part in myocardial injury and repair. (11)(12) We found that there was a significant correlation between abnormal hs-cTnl values and D-dimer values, rather than other inflammatory parameters. D-dimer is a soluble fibrin degradation product that results from ordered breakdown of thrombi by the coagulative and fibrinolytic system. (13) Recently, Tang $N$ etc. found the non-survivors revealed significantly higher D-dimer compared to survivors on admission outcomes of consecutive 183 patients with confirmed COVID-19. (14) These all pointed to high hs-cTnI values more likely accompanied by bad prognosis in COVID-19 patients. What's more, patients in male group and 60-79 groups tend to develop higher hs-cTnl values than female and other age groups, which was in consistent with the overall high proportion of elderly male non-survivors. Also, longitudinal analysis showed that of the hs-cTnl values could increase more than one week before death. We believed the indicator hs-cTnl might be underestimated during the COVID-19 development.

There are several limitations in this study. This is a single center analysis which might lead to case selection bias, especially as Sino-French Branch of Tongji Hospital is designated for treatments of severe patients with COVID-19 by the government. Most patients were transferred from other hospitals and early clinical medical records were briefly documented or unavailable. Finally, age, sex, disease severity matched survival groups were not included in this study, which could provide more valuable information of risk factors for prognosis.

\section{Conclusions}

Most non-survivors with SARS-CoV-2 infection were old with chronic illnesses. Abnormal elevation of hscTnl accompanied with over activated inflammatory factors and depressed lymphocytes was commonly observed in death patients with SARS-CoV-2 infection, which may play an important role in risk stratification and prognostic prediction.

\section{Abbreviations}


SARS-CoV-2, Severe acute respiratory syndrome coronavirus 2

COVID-19, Coronavirus disease 2019

CT, Computed tomography

ALT, Alanine aminotransferase

AST, Aspartate aminotransferase

ESR, Erythrocyte sedimentation rate

hs-CRP, High sensitive C-reactive protein

LDH, Lactate dehydrogenase

hs-cTnl, High sensitive cardiac troponin I

NT-proBNP, amino-terminal pro-brain natriuretic peptide

IL, interleukin

TNF-a, tumor necrosis factor-a.

ECMO, Extracorporeal membrane oxygenation

CRRT, Continuous renal replacement therapy

\section{Declarations}

\section{Ethics approval and consent to participate}

This retrospective study was approved by the institutional review board of Tongji Hospital of Huazhong University of Science and Technology.

\section{Consent for publication}

Written informed consent was waived due to the emergency outbreak of COVID-19.

\section{Availability of data and materials}

The datasets used and/or analysed during the current study are available from the corresponding author on reasonable request.

\section{Competing interests}

The authors declare that they have no competing interests. 


\section{Funding}

This study received no funding.

\section{Authors' contributions}

All authors had access to the data and contributed to writing this manuscript. Conceived and designed the experiments: Rong Liu, Ke Yao, Yin Zhao. Performed the study: Ke Yao, Yin Zhao, Xiangtian Xiao, GuiyunWu, Ruxin Xie, Yali Wang, Jun Hu, He xie Cai. Analyzed the data: Ke Yao, Yin Zhao, Xiangtian Xiao, GuiyunWu, Rong Liu. Contributed materials/analysis tools: Rong Liu, Ruxin Xie, Yali Wang, Jun Hu, He xie Cai.

\section{Acknowledgements}

Not applicable.

\section{References}

1.Walls AC, Park YJ, Tortorici MA, et al. Structure, Function, and Antigenicity of the SARS-CoV-2 Spike Glycoprotein. Cell. 2020.

2.Wang YX, Wang YY, Chen Y, et al. Unique epidemiological and clinical features of the emerging 2019 novel coronavirus pneumonia (COVID-19) implicate special control measures. J MED VIROL. 2020

3.Yan L. Xia LM. Coronavirus Disease 2019 (COVID-19): Role of Chest CT in Diagnosis and Management. AJR Am J Roentgenol. 2020; 215:1-7

4. WHO. Clinical management of severe acute respiratory infection when Novel coronavirus (nCoV) infection is suspected: interim guidance. January, 2020. https://www.who.int/internal-publicationsdetail/clinical-management-of-severeacute-respiratory-infection-when-novel-coronavirus-(ncov)-infectionis-suspected

5. Westwood M, Van Asselt T, Ramaekers B, et al. High-sensitivity troponin assays for the early rule-out or diagnosis of acute myocardial infarction in people with acute chest pain: a systematic review and costeffectiveness analysis. Health Technol Assess. 2015, 19(44):1-234.

6. Guan WJ, Ni ZY, Hu Y, et al. Clinical Characteristics of Coronavirus Disease 2019 in China. N Engl J Med. 2020

7. Yang $X, Y u$ Y, Xu J, et al. Clinical course and outcomes of critically ill patients with SARS-CoV-2 pneumonia in Wuhan, China: a single-centered, retrospective, observational study. Lancet Respir Med. 2020 
8. Chen C, Chen C, Yan JT, et al. Analysis of myocardial injury in patients with COVID-19 and association between concomitant cardiovascular diseases and severity of COVID-19. Zhonghua Xin Xue Guan Bing Za Zhi 2020;48:E008.

9. Lee CC, Huang SS, Yeo YH, et al. High-sensitivity-cardiac troponin for accelerated diagnosis of acute myocardial infarction: A systematic review and meta-analysis. Am J Emerg Med. 2019

10. Rahman F, Zhang 9.Z, Zhao D, et al. Association of High-Sensitivity Troponin with Cardiac CT Angiography Evidence of Myocardial and Coronary Disease in a Primary Prevention Cohort of Men: Results from MACS. J Appl Lab Med.2019; 4:355-369.

11. Frangogiannis, N.G. The inflammatory response in myocardial injury, repair, and remodelling. Nat Rev Cardiol, 2014, 11(5):255-265.

12. Carrillo-Salinas FJ, Ngwenyama N, Anastasiou M, et al. Heart Inflammation: Immune Cell Roles and Roads to the Heart. Am J Pathol. 2019;189:1482-1494.

13. Weitz JI, Fredenburgh JC. A Test in Context: D-Dimer.J Am Coll Cardiol. 2017; 70:2411-2420.

14. Tang N, Li D, Wang X. Abnormal Coagulation parameters are associated with poor prognosis in patients with novel coronavirus pneumonia. J Thromb Haemost. 2020

\section{Tables}

Table 1. Demographics characteristics and symptoms of non-survival patients with COVID-19 on admission

\begin{tabular}{llll}
\hline Characteristics & Total $(\mathrm{n}=194)$ & Symptoms & Total $(\mathrm{n}=192)$ \\
\hline Age (y), means \pm SD & $69.62 \pm 10.98$ & Fever & $160(83 \%)$ \\
Age range, years & $36-95$ & Cough & $133(69.3 \%)$ \\
$\quad 35-49$ & $6(3.1 \%)$ & Malaise & $58(30.2 \%)$ \\
$\quad 50-59$ & $28(14.4 \%)$ & Chills or fatigue & $27(14.1 \%)$ \\
$60-69$ & $61(31.4 \%)$ & Dyspnea & $126(65.6 \%)$ \\
$\quad 70-79$ & $60(30.9 \%)$ & Rhinorrhea & $3(1.6 \%)$ \\
$\quad 390$ & $39(20.1 \%)$ & Myalgia & $28(14.9 \%)$ \\
Sex & & Chest pain & $3(1.6 \%)$ \\
Female & $61(31.4 \%)$ & Poor appetite & $16(8.3 \%)$ \\
Male & $133(68.6 \%)$ & Vomiting & $39(20.3 \%)$ \\
Chronic medical illness & & Diarrhea & $18(9.4 \%)$ \\
Not clear & $21(10.8 \%)$ & Abdominal pain & $5(2.6 \%)$ \\
None & $28(14.4 \%)$ & Headache & $4(2.1 \%)$ \\
Yes & $145(74.7 \%)$ & Consciousness disorder & $14(7.3 \%)$ \\
Hypertension & $92(63.4 \%)$ & Palpitation & $5(2.6 \%)$ \\
Diabetes & $40(27.5 \%)$ & Hemoptysis & $4(2.1 \%)$ \\
Cardiovascular disease & $39(26.9 \%)$ & Pharyngalgia & $3(1.6 \%)$ \\
Chronic pulmonary & $18(12.4 \%)$ & Night sweat & $4(2.1 \%)$ \\
disease & $10(6.9 \%)$ & Hematochezia & $1(0.5 \%)$ \\
Hepatitis & $9(6.2 \%)$ & Incontinence & $1(0.5 \%)$ \\
Cerebrovascular disease & $9(6.2 \%)$ & & \\
Malignancy & $9(6.2 \%)$ & & \\
Chronic kidney disease & $2(1.4 \%)$ & & \\
Major surgery for last 3 months & $14(9.7 \%)$ & & \\
Others & $66(45.5 \%)$ & & \\
$\geq 2$ types & & & \\
& & & \\
\hline
\end{tabular}


Notes: Other chronic medical illnesses included HIV infection, pancreatitis, gastric ulcer, rheumatoid arthritis, cholelithiasis cholecystectomy, varicose veins of the lower extremities, operation history of appendicitis, lumbar disc protrusion, hyperlipidemia, etc..

Table 2. Last recorded laboratory findings of death patients with COVID-19

\begin{tabular}{|c|c|c|c|}
\hline Median (range) & Total & Abnormal & means $\pm \mathrm{SD}$ \\
\hline White blood cell count, $\times 10^{9} / \mathrm{L}$ & 185 & $143 \square 77.3 \% \square$ & $13.47 \pm 7.13$ \\
\hline Neutrophil, $\times 10^{9} / \mathrm{L}$ & 185 & $156 \square 84.3 \% \square$ & $12.84 \pm 8.56$ \\
\hline Lymphocyte count, $\times 10^{9} / \mathrm{L}$ & 185 & $169 \square 91.4 \% \square$ & $0.58 \pm 0.42$ \\
\hline Platelet count, $\times 10^{9} / \mathrm{L}$ & 185 & $121 \square 65.4 \% \square$ & $119.20 \pm 91.95$ \\
\hline $\mathrm{ALT}, \mathrm{U} / \mathrm{L}$ & 184 & 81ロ44.0\%॰ & $53.53 \pm 100.70$ \\
\hline $\mathrm{AST}, \mathrm{U} / \mathrm{L}$ & 184 & $121 \square 65.4 \% \square$ & $101.70 \pm 240.30$ \\
\hline D-dimer, mg/L & 178 & $176 \square 98.9 \% \square$ & $12.04 \pm 9.04$ \\
\hline ESR, mm/H & 126 & 88ロ69.8\%ロ & $47.83 \pm 44.85$ \\
\hline hs-CRP, mg/L & 168 & $168 \square 100 \% \square$ & $129.30 \pm 84.36$ \\
\hline $\mathrm{LDH}, \mathrm{U} / \mathrm{L}$ & 160 & $155 \square 96.9 \% \square$ & $732.50 \pm 445.70$ \\
\hline Blood urea nitrogen, $\mathrm{mmol} / \mathrm{L}$ & 180 & $138 \square 76.7 \% \square$ & $17.20 \pm 13.73$ \\
\hline Creatinine, $\mu \mathrm{mol} / \mathrm{L}$ & 168 & $101 \square 60.1 \% \square$ & $175.4 \pm 201.9$ \\
\hline hs-cTnI, pg/mL & 170 & $141 \llbracket 82.9 \% \square$ & $1591 \pm 6229$ \\
\hline NT-proBNP, pg/mL & 157 & $143 \square 91.1 \% \square$ & $6695 \pm 12858$ \\
\hline $\mathrm{IL}-1 \beta, \mathrm{pg} / \mathrm{mL}$ & 115 & 24ロ20.9\%ロ & $6.55 \pm 5.32$ \\
\hline $\mathrm{IL}-2 \mathrm{R}, \mathrm{U} / \mathrm{mL}$ & 112 & 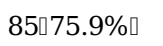 & $1286.0 \pm 932.7$ \\
\hline IL-6, pg/mL & 112 & 111־99.1\%ם & $524.6 \pm 1192.0$ \\
\hline IL-8, pg/mL & 113 & 39ロ34.5\%ロ & $227.8 \pm 865.0$ \\
\hline IL-10, pg/mL & 111 & 67ロ60.4\%ロ & $28.29 \pm 61.81$ \\
\hline TNF- $\alpha, p g / m L$ & 112 & 87๑77.7\%ロ & $19.62 \pm 20.77$ \\
\hline
\end{tabular}

Notes: SD, standard deviation. ALT, alanine aminotransferase; AST, aspartate aminotransferase; ESR, erythrocyte sedimentation rate; hs-CRP, high sensitive C-reactive protein; LDH, lactate dehydrogenase; hs-cTnI, high sensitive cardiac troponin I; NT-proBNP, amino-terminal pro-brain natriuretic peptide; IL, interleukin; TNF- $\alpha$, tumor necrosis factor- $\alpha$.

Table 3. Complications and treatments of death patients with COVID-19 


\begin{tabular}{ll}
\hline Complications & Total $(\mathrm{n}=110)$ \\
\hline ARDS & $69(62.7 \%)$ \\
Cardiac injury & $50(45.4 \%)$ \\
Arrhythmia & $11(10.0 \%)$ \\
Acute kidney injury & $16(14.5 \%)$ \\
Liver dysfunction & $6(5.5 \%)$ \\
Septic shock & $10(9.1 \%)$ \\
Secondary infection & $3(2.7 \%)$ \\
DIC & $13(11.8 \%)$ \\
Brain injury & $4(3.6 \%)$ \\
Others & $4(3.6)$ \\
MODS & $56(50.9 \%)$ \\
Treatments & Total (n=194) \\
Oxygen therapy & $131(67.5 \%)$ \\
Mechanical ventilation & $157(80.9 \%)$ \\
Invasive & $95(49.0 \%)$ \\
Noninvasive & $62(31.90 \%)$ \\
Antivirus medication & $136(70.1 \%)$ \\
Antibacterial medication & $170(87.6 \%)$ \\
Antifungal medication & $0(0.0 \%)$ \\
Systemic glucocorticoids & $159(90.0 \%)$ \\
Intravenous immune globulin & $79(40.7 \%)$ \\
ECMO & $2(1.0 \%)$ \\
CRRT & $4(2.1 \%)$ \\
\hline
\end{tabular}

ARDS, acute respiratory distress syndrome; DIC, disseminated intravascular coagulation; MODS, multiple organ dysfunction syndrome; ECMO, extracorporeal membrane oxygenation; CRRT: continuous renal replacement therapy

Figures 


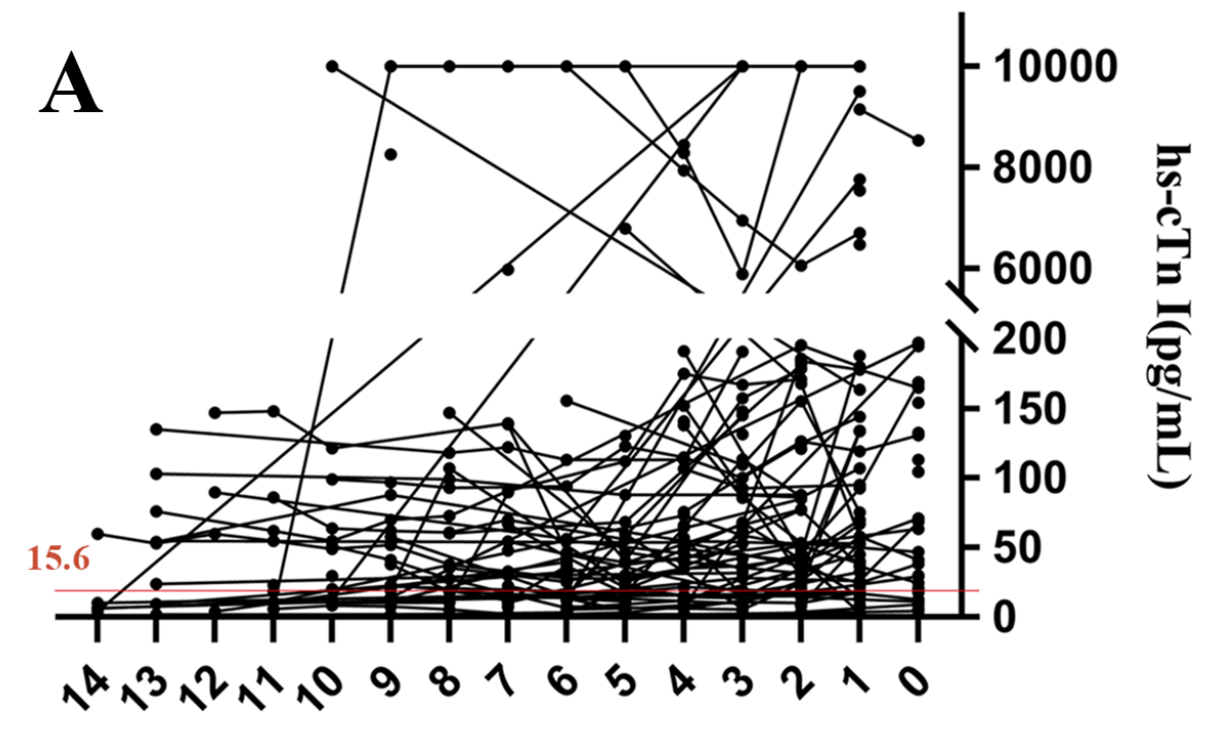

Days before death

B
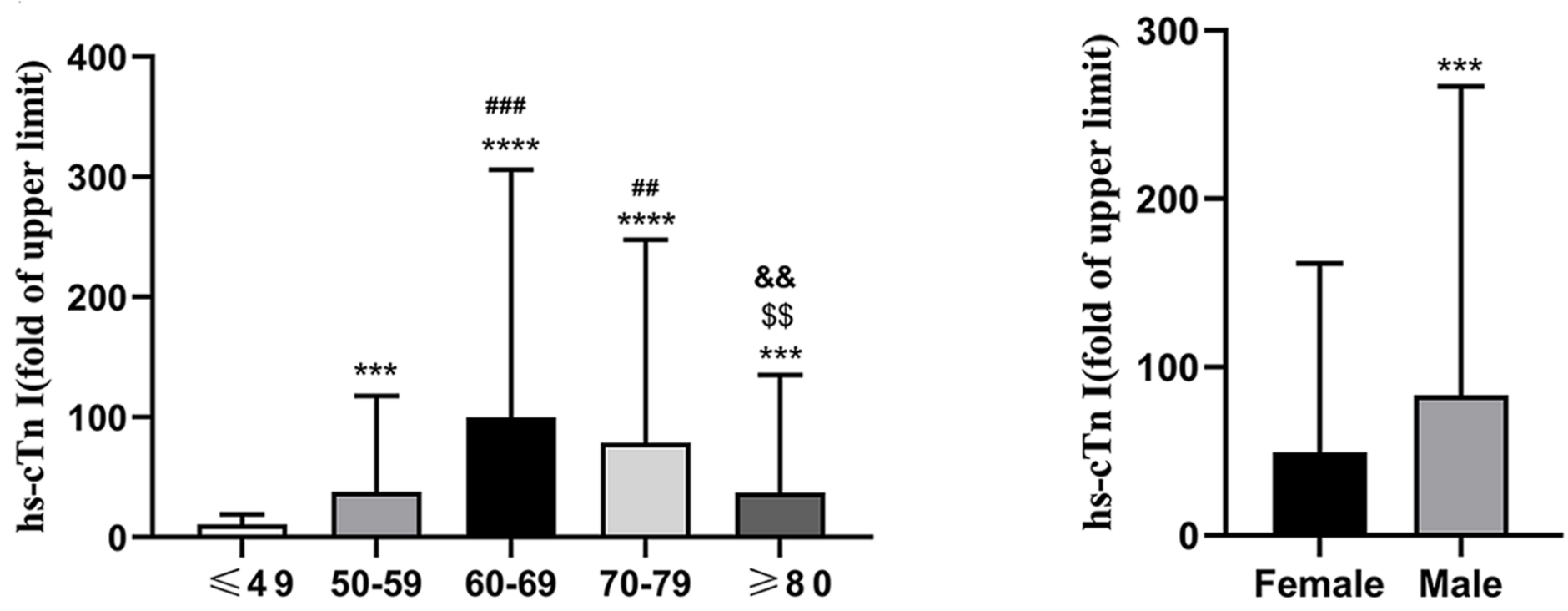

Female Male

Age(y)

Figure 1

The hs-cTnl levels of the patients with COVID-19 14 days before death and sex and age differences Notes: A: The hs-cTnl values of the patients 14 days before death. B: The hs-cTnl level (fold of upper limit) in different age groups. *, compared to the group age $\leq 49 ; \#$, compared to group 50-59; $\$$,

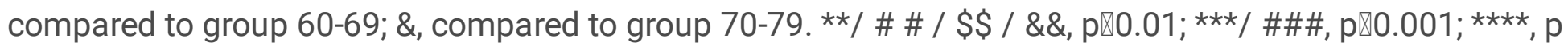
$₫ 0.0001$. C: The hs-cTnl level (fold of upper limit) in different sex groups. ${ }^{\star \star}, \mathrm{p} \otimes 0.001$. Data were given as mean \pm standard deviation. Unpaired $t$ test was used(two-tailed). A $p$ value less than 0.05 was considered statistically significant. 


\section{Supplementary Files}

This is a list of supplementary files associated with this preprint. Click to download.

- SupplementaryTables.docx 